\title{
Is adding fluoroquinolones to regimens for treating isoniazid-resistant tuberculosis necessary?
}

\author{
Roland Diel ${ }^{1,2}$ and Neil W. Schluger ${ }^{3}$ \\ Affiliations: ${ }^{1}$ Institute for Epidemiology, University Medical Hospital Schleswig-Holstein, Kiel, Germany. \\ ${ }^{2}$ Lungenclinic Grosshansdorf, Airway Research Center North (ARCN), Member of the German Center for Lung \\ Research (DZL), Großhansdorf, Germany. ${ }^{3}$ Division of Pulmonary, Allergy and Critical Care Medicine, \\ Columbia University Medical Center, New York, NY, USA.
}

Correspondence: Roland Diel, Institute for Epidemiology, University Medical Hospital Schleswig-Holstein, Niemannsweg 11, 24105 Kiel, Germany. E-mail: roland.dieldepi.uni-kiel.de

@ERSpublications

In patients with isoniazid-resistant tuberculosis, administering fluorochinolones may not be required if the duration of $(\mathrm{H}) \mathrm{RZE}$ treatment is long enough (12 months), even with relatively short durations of $\mathrm{Z}$ in the initiation phase http://bit.ly/2NjsCEQ

Cite this article as: Diel R, Schluger NW. Is adding fluoroquinolones to regimens for treating isoniazidresistant tuberculosis necessary? Eur Respir J 2019; 54: 1901494 [https://doi.org/10.1183/13993003.014942019].

Resistance against first-line drugs for treating tuberculosis (TB) continues to be a major public health challenge that hampers the World Health Organization (WHO)'s End TB strategy [1]. The End TB strategy aims to reduce TB deaths by $95 \%$ and new TB cases by $90 \%$ between 2015 and 2035. In 2017, the WHO recorded 558000 cases (range 483000-639000) of TB not treatable with rifampicin, the most effective first-line anti-TB drug. Of these, $82 \%$ cases were of multidrug-resistant TB (MDR-TB) [2].

Worldwide, however, the most common form of drug-resistant TB is isoniazid-resistant, rifampicinsusceptible tuberculosis. In 1998, the WHO published the results of a global survey of drug-resistant TB, performed in 35 countries or regions on five continents between 1994 and 1997 [3]. At that time, isoniazid mono-resistance had a prevalence of $7.3 \%$. According to the most recent best estimates from 149 countries over the period 2003-2017 [2], isoniazid resistance without concurrent rifampicin resistance is still present in $7.1 \%$ (95\% CI $6.2-8.0 \%$ ) of new TB cases and in $7.9 \%$ (95\% CI $5.9-10 \%$ ) of previously treated TB patients. The distribution of isoniazid resistance may vary substantially by country, but it is particularly concentrated in the countries of the former Soviet Union, where the global figures have more than doubled to $16.1 \%$ of TB cases [4].

The treatment of isoniazid-resistant TB, not only that of MDR-TB, requires particular attention: as pointed out in a meta-analysis by Menzies et al. [5] drawing from 57 randomised controlled trials, the incidence rates of treatment failure associated with isoniazid resistance are 10.9 times higher, the relapse rates 1.8 fold higher and rates of acquired drug resistance 5.1 times higher than in cases of fully susceptible TB.

In response to the overall growing problem of drug-resistant $\mathrm{TB}$, the $\mathrm{WHO}$ issued consolidated guidelines of which one, dealing with the current evidence for the treatment of isoniazid resistance, was published in 2018 [6]. The reviewers could not trace cohort or randomised controlled trials that included fluoroquinolones as part of standardised TB regimens designed primarily for isoniazid $(\mathrm{H})$-resistant $\mathrm{TB}$, so the datasets of only 251 patients in 15 observational studies who received a fluoroquinolone (Fq) for at 
least 1 month in addition to at least 6 months of rifampicin (R), ethambutol (E) and pyrazinamide $(\mathrm{Z})$ could be included and compared to 1350 patients without fluoroquinolone. Propensity score matching was performed, and the 251 patients receiving fluoroquinolone had significantly higher odds of treatment success (adjusted odds ratio (aOR) 2.8, 95\% CI 1.1-7.3) than patient who did not. No difference, however, was found between the two groups with respect to mortality (aOR 0.7, 95\% CI 0.4-1.19). The median duration of use of fluoroquinolones in $\geqslant 6$ months $(H)$ REZFq regimens was 6.1 months (interquartile range 3.5-8.4 months); of rifampicin 9.0 months (7.2-11.1 months); of ethambutol 9.0 months (7.3-11.1 months); and of pyrazinamide 8.9 months (6.8-10.7 months).

Based on those data, which were later included in a meta-analysis published in the Lancet Respiratory Medicine [7], the use of a fluoroquinolone, restricted to levofloxacin (Lfx), in patients with isoniazid-resistant TB was recommended; specifically, a dose of 750-1000 mg in a REZ regimen (with or without isoniazid) for at least 6 months. However, as the overall quality of the studies according to the GRADE methodology was rated as low to very low for all regimens, these recommendations were classified as "conditional".

Indeed, while current evidence for treating isoniazid resistance appears to be insufficient, the existing guidelines on the topic in the USA and Europe are remarkably divergent: the 2003 recommendations of the American Thoracic Society propose a 6-month regimen of RZE, with fluoroquinolone added only in extensive disease [8]. For the UK, the guidelines of the National Institute for Health and Care Excellence recommends a 9-month regimen of 2 months RZE, followed by 7 months of RE. They further recommend considering extending treatment to 10 months in cases of extensive TB disease [9]. Whereas the Swiss guidelines refer to the conditional WHO recommendations [10], the German guidelines recommend a brief (2-month) treatment that includes $\mathrm{Z}$ (RZEFq) to be followed by REFq for a further 4-7 months [11].

It is in this context that the work of STAGG et al. [12] in this issue of the European Respiratory Journal should be viewed. They describe a retrospective cohort study of 626 notified cases of isoniazid-resistant TB in patients treated in 30 hospitals in London, UK, between 2009 and 2013. In their paper, the authors present the results of 594 individuals of whom 330 (55.6\%) were treated with (H)RfZE (Rf refers to rifamycins) and 211 (35.5\%) with (H)RfZE-Fq. The median overall treatment period was 11.9 months and median pyrazinamide treatment duration 2.1 months. In a univariable logistic regression model comparing a (H)RfZE-strategy with and without fluoroquinolone, predominately moxifloxacin, there was no difference between the two regimens in the odds of a negative overall outcome (OR 1.05, 95\% CI 0.60-1.82). The non-significance figure did not change in the final multivariable logistic regression model, which included only 435 patients, and yielded an adjusted odds ratio of 0.99 (95\% CI 0.53-1.85).

What can we learn from this study? The findings of STAGG et al. [12] demonstrate that, if the duration of (H)RZE treatment is long enough (12 months), a fluoroquinolone may not be required, even with relatively short durations (median 2 months in the initiation phase) of pyrazinamide. Apparently that treatment was powerful enough to overcome the effect of resistance mutations in the patients' TB strains. The composite, however, could not be fully evaluated. Whole genome sequencing (WGS), which did not become routine in the UK until 2012-2013, makes it possible to characterise the highly variable collection of gene mutations that lead to resistance. WGS data was available to STAGG et al. [12] for only 161 of the $626(25.7 \%)$ of the cases they studied, i.e. those from the end of the collection period.

As the $\geqslant 6(\mathrm{H})$ RZE-6Lfx option entails a long period of waiting (minimum of 1.5 or up to 3 months) for the results of phenotypical drug susceptibility testing, its "6-month" characterisation is misleading. The regimen, in fact, requires a minimum of 7.5 or up to 9 months, because the 6-month treatment with a fluoroquinolone can start only when the drug susceptibility data is received. Hence the 12-month $(\mathrm{H}) \mathrm{RZE}$ treatment should not be considered to take twice as long as the WHO-recommended treatment, but rather 3-4.5 months longer. This of course could change in some high-resource settings, if WGS replaces phenotypic drug susceptibility testing.

The study is limited by the fact that it is a retrospective cohort study, not a randomised controlled trial, and may therefore have been subject to selection bias and imperfect information on drug intake. The authors suggest that the 12-month regimen without administering a fluoroquinolone may result in fewer adverse events than experienced under the WHO recommendations, but they do not report the type and frequency of events occurring in their cohort of patients. Therefore, a translation of adverse events into cost figures for the purpose of a cost-benefit comparison of the two regimens remains reserved for future trials.

In any case, the study by STAGG et al. [12] provides very useful assistance for treating those patients who have contraindications for treatment with fluoroquinolone, especially for patients with kidney disease or mental illness. Also, fluoroquinolone interaction is known to place older persons on long-standing glucocorticoid therapy at higher risk of tendon damage [13]. Additional data from randomised trials to 
better characterise the benefit-harm profile of fluoroquinolone is desirable in the long term. A large phase two trial in which moxifloxacin was substituted for isoniazid in the induction phase of chemotherapy provided evidence that such a regimen (FqRZE) results in equivalent 2-month culture conversion rates as HRZE [14]. Whether this would translate into equal effectiveness of a full 6-month fluoroquinolonecontaining regimen remains to be tested. In the meantime, the findings of STAGG et al. [12] are very welcome at present for enhancement of the current guidelines of treating drug-resistant TB.

Conflict of interest: R. Diel reports grants and personal fees from Bayer Vital, outside the submitted work. N.W. Schluger has nothing to disclose.

\section{References}

1 World Health Organization (WHO). End TB Strategy www.who.int/tb/post2015_strategy/en/ Date last accessed: 21 July 2019.

2 World Health Organization (WHO). Global Tuberculosis Report 2018. www.who.int/tb/publications/global_ report/en/ Date last accessed: 21 July 2019.

3 Pablos-Mendez A, Raviglione MC, Laszlo A, et al. Global surveillance for antituberculosis-drug resistance, 19941997. World Health Organization-International Union against Tuberculosis and Lung Disease Working Group on Anti-Tuberculosis Drug Resistance Surveillance. N Engl J Med 1998; 338: 1641-16494.

4 Stagg HR, Lipman MC, McHugh TD, et al. Isoniazid-resistant tuberculosis: a cause for concern? Int J Tuberc Lung Dis 2017; 21: 129-139

5 Menzies D, Benedetti A, Paydar A, et al. Effect of duration and intermittency of rifampin on tuberculosis treatment outcomes: a systematic review and meta-analysis. PLoS Med 2009; 6: e1000146.

6 World Health Organization. WHO Treatment Guidelines for Isoniazid-resistant Tuberculosis: Online Annexes. Supplement to the WHO Treatment Guidelines for Drug-resistant Tuberculosis. www.who.int/tb/publications/ 2018/WHO_guidelines_isoniazid_resistant_TB/en/ Date last accessed: 21 July 2019. Last updated July 2018.

7 Fregonese F, Ahuja SD, Akkerman OW, et al. Comparison of different treatments for isoniazid-resistant tuberculosis: an individual patient data meta-analysis. Lancet Respir Med 2018; 6: 265-275.

8 Blumberg HM, Burman WJ, Chaisson RE, et al. American Thoracic Society/Centers for Disease Control and Prevention/Infectious Diseases Society of America: treatment of tuberculosis. Am J Respir Crit Care Med 2003; 167: 603-662.

9 National Institute for Health and Care Excellence. Tuberculosis. www.nice.org.uk/guidance/ng33/chapter/ recommendations\#drugresistant-tb-excluding-multidrug-and-extensively-drugresistant-tb Date last accessed: 21 July 2019. Date last updated: 9 Jan 2016.

10 Tuberculosis in Switzerland. Guide for Health Professionals, Version 3.2019. www.tbinfo.ch/fileadmin/user upload/1wissenszentrum/Publikationen/Handbuch_Tuberkulose/Handbuch_TB_DE_16_05_19.pdf Date last accessed: 21 July 2019

11 Schaberg T, Bauer T, Brinkmann F, et al. [Tuberculosis Guideline for Adults - Guideline for Diagnosis and Treatment of Tuberculosis including LTBI Testing and Treatment of the German Central Committee (DZK) and the German Respiratory Society (DGP)]. Pneumologie 2017; 71: 325-397.

12 Stagg HR, Bothamley GH, Davidson JA, et al. Fluoroquinolones and isoniazid resistant tuberculosis: implications for the 2018 WHO guidance. Eur Respir J 2019; 54: 1900982.

13 U.S. Food and Drug Administration. Drug Safety Communication: FDA updates warning for fluoroquinolone antibiotics on risks of mental health and low blood sugar adverse reactions. Jul 10, 2018. www.fda.gov/newsevents/press-announcments/fda.updates-warnings-fluoroquinolone-antobiotics-risks-mental-health-and-low-bloodsugar-adverse. Date last accessed: July 21, 2019.

14 Dorman SE, Johnson JL, Goldberg S. Substitution of moxifloxacin for isoniazid during intensive phase treatment of pulmonary tuberculosis. Am J Respir Crit Care Med 2009; 180: 273-280. 